\title{
ATLAS DE PÓleN E ESPOROS DO VALE DO RIO CAÍ, RS, BRASIL ${ }^{1}$
}

\author{
Soraia Girardi Bauermann ${ }^{2}$, Andreia Cardoso Pacheco Evaldt ${ }^{2}$ e Sabrina Castelo Branco ${ }^{2}$
}

\begin{abstract}
RESUMO - O atlas descritivo de polens e esporos de plantas do Vale do Rio Caí tem o objetivo de facilitar a comparação com os palinomorfos dispersos nos sedimentos fósseis e fornecer dados para ações de manejo ambiental. O atlas consta de 93 espécies características das quatro formações vegetacionais do Vale do Rio Caí, incluindo Floresta Ombrófila Mista, Floresta Estacional Decidual, Floresta Estacional Semidecidual e Estepe, além de plantas exóticas e de locais alterados. Incluíram-se, nessa primeira parte, descrições detalhadas e fotomicrografias de 24 espécies, 21 gêneros e 17 famílias.
\end{abstract}

Palavras-chave: Pólen, esporos e Vale do Rio Caí.

\section{POLLEN AND SPORES ATLAS OF THE CAÍ RIVER VALLEY, RS, BRAZIL}

\begin{abstract}
A descriptive atlas of pollen and spores from plants of Caí river Valley aimed to facilitate the identification of dispersed palynomorphs in fossil sediments and provide data for environmental management actions. The atlas presents 93 characteristic species of four Caí river valley plant associations, including Floresta Ombrófila Mista, Floresta Estacional Decidual, Floresta Estacional Semidecidual and Estepe, besides exotic plants and disturbed sites. In this first part, detailed descriptions and light micrographs of 24 species, 21 genera, and 17 families were included.
\end{abstract}

Keywords: Pollen, spores and Caí River Valley.

\section{INTRODUÇÃO}

A fragmentação da paisagem é uma das principais alterações ambientais causadas pela atividade humana e acarreta, como consequência, a contínua perda da biodiversidade. Ações de manejo e uso sustentável devem estar fundamentadas em informações biológicas de amplo espectro, como biologia floral e reprodutiva, fenologia, dinâmica e genética de populações, fluxo gênico e dispersão de sementes, entre outros.

Há, no entanto, carência de dados acerca da morfologia polínica que possam subsidiar estudos de fluxo gênico realizado via pólen, quer seja por anemofilia, quer seja por zoofilia. Além do mais, o conhecimento da flora polínica atual serve de paradigma para estudos de dinâmica vegetacional, pois conjuntos polínicos semelhantes (atuais e passados) indicam, por analogia, condições ambientais similares.
O Vale do Rio Caí fica ao norte de Porto Alegre, entre o planalto brasileiro e a depressão central. Compreende, ao longo do seu curso d'água, uma extensão de $275,6 \mathrm{~km}$. As formações fitoecológicas predominantes na região são: Floresta Ombrófila Mista(FOM), Floresta Estacional Decidual (FED), Floresta Estacional Semidecidual (FES) e Estepe, que, por vezes, podem estar entrecortadas por Florestas de Galeria (SEPLAN/IBGE, 1986).

Levantamento botânico prévio foi realizado através de estudo fitossociológico no Vale do Rio Caí, desde suas nascentes até as proximidades da foz, abrangendo o curso principal e seus contribuintes (Figura 1), em que houve o registro da ocorrência de 747 espécies distribuídas em 414 gêneros e 129 famílias, sendoAmaranthaceae, Asteraceae, Euphorbiaceae e Myrtaceae as famílias com maior riqueza de espécies. Durante este estudo foram elaboradas exsicatas do material-testemunha dos principais espécimes das formações vegetacionais da região (DIESEL, 2005).

\footnotetext{
${ }^{1}$ Recebido em 30-01-2008 e aceito para publicação em 23.06.2009.

${ }^{2}$ Universidade Luterana do Brasil - Campus Canoas, RS - Brasil. E-mail: <soraia.bauermann@ulbra.br〉.
} 


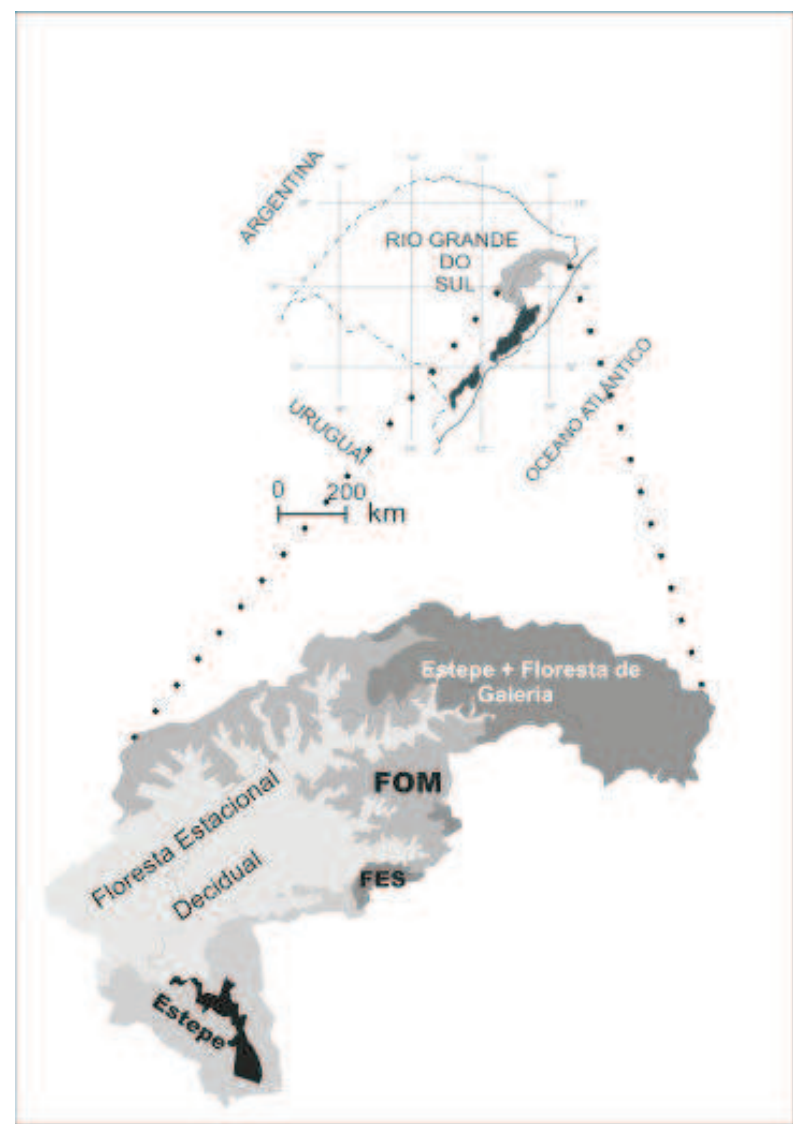

Figura 1 - Mapa de localização da área estudada e suas comunidades vegetacionais. FOM (Floresta Ombrófila Mista) e FES (Floresta Estacional Semidecidual).

Figure 1-Map of location of the studied area and its vegetation communities. FOM (Floresta Ombrófila Mista) and FES (Floresta Estacional Semidecidual).

O estudo teve como objetivo principal a elaboração de um atlas da diversidade polínica no Vale do Rio Caí, em material polinífero extraído de plantas nativas férteis daquela coleção. Foram adicionadas ao atlas algumas espécies exóticas comuns naquela região, como Ligustrum sinense e Ricinus communis.

O atlas apresenta 93 espécies, e nessa primeira parte são descritos 22 grãos de pólen e dois esporos.

\section{MATERIAL E MÉTODOS}

O material polínico fértil foi coletado a partir de exsicatas depositadas no Herbário do Museu de Ciências da Universidade Luterana do Brasil
(HERULBRA). Foram coletados materiais férteis de 93 espécies, sendo essas amostras processadas pela técnica de acetólise, segundo Erdtman (1952). As lâminas permanentes foram preparadas utilizando-se como meio de montagem gelatina glicerinada. Foram confeccionadas cinco lâminas permanentes para cada espécie, as quais se encontram depositadas na palinoteca do Laboratório de Palinologia da ULBRA. A análise dos grãos de pólen foi realizada em microscópio óptico modelo Leica DMLB, com aumento de 1.000x. Os grãos foram descritos quanto a unidade polínica, tamanho, âmbito, forma, número de aberturas, ornamentação $(\mathrm{O})$ e espessura (Ex) da exina. As medidas realizadas no máximo uma semana após o processo de acetólise foram feitas utilizando-se em média, 25 grãos. Para a descrição do tamanho dos grãos, foi utilizada a média aritmética das medidas do diâmetro polar/equatorial (P/E), segundo Erdtman (1952). Nos grãos esféricos foi medido o diâmetro (D); nas políades e tétrades foram medidos os diâmetros maior (DM) e menor (Dm), em vista frontal. Para as descrições polínicas foi utilizada a nomenclatura proposta por Barth e Melhem (1988), e complementada por Punt et al. (2007), bem como literatura especializada: SalgadoLaboriau (1973), Roubik e Moreno (1991), Lorscheitter et al. (1998), Colinvaux at al. (1999), Melhem et al. (2003), Neves e Bauermann (2003), Neves et al. (2003), Willard et al. (2004) e Willberger et al. (2004).

As descrições completas estão organizadas por família botânica em ordem alfabética, iniciando-se com pteridófitos, seguidos das angiospermas. A nomenclatura botânica foi consultada em banco de dados e literatura especializada (IPNI, 2007; MOBOT, 2007). As fotomicrografias nas figuras estão organizadas levando-se em consideração a diferença proporcional de dimensões entre os grãos de pólen para facilitar a sua identificação.

\section{RESULTADOS}

Foram descritos, nessa primeira parte, 24 esporomorfos distribuídos entre pteridófitos e angiospermas, constituintes de várias formações ecológicas (Tabela 1). Os pteridófitos estão representados por duas famílias, dois gêneros e duas espécies. Entre as angiospermas, 15 famílias, 19 gêneros e 22 espécies foram registrado. 
Tabela 1 - Táxons incluídos no estudo polínico e comunidades vegetacionais nas quais eles ocorrem. Os dados apresentados foram compilados de Seplan/IBGE (1986), Backes e Nardino (2003), Sobral et al. (2006) e Lopes e Gonçalves (2007). FOM (Floresta Ombrófila Mista), FED (Floresta Estacional Decidual) e FES (Foresta Estacional Semidecidual).

Table 1 - Taxa included in the pollinic study and vegetable communities in which they occur. The data were compiled from Seplan/IBGE (1986), Backes and Nardino (2003), Sobral et al. (2006) and Lopes and Gonçalves (2007). FOM (Floresta Ombrófila Mista), FED (Floresta Estacional Decidual) and FES (Foresta Estacional Semidecidual).

\begin{tabular}{|c|c|c|c|c|c|c|}
\hline Família & Espécie & FOM & FED & FES & Estepe & Exóticas \\
\hline Berberidaceae & Berberis laurina & $\mathrm{X}$ & & & & \\
\hline Caprifoliaceae & Sambucus australis & & $\mathrm{X}$ & & & \\
\hline Celastraceae & Maytenus ilicifolia & $\mathrm{X}$ & & $\mathrm{X}$ & $\mathrm{X}$ & \\
\hline Ericaceae & Agarista eucalyptoides & $\mathrm{X}$ & & $\mathrm{X}$ & & \\
\hline Erythroxylaceae & Erythroxylum argentinum & & & & $\mathrm{X}$ & \\
\hline Escalloniaceae & Escallonia megapotamica & $\mathrm{X}$ & $X$ & $\mathrm{X}$ & & \\
\hline Flacourtiaceae & Casearia sylvestris & $\mathrm{X}$ & $x$ & $\mathrm{X}$ & $\mathrm{X}$ & \\
\hline Melastomataceae & Miconia hyemalis & $\mathrm{X}$ & & & & \\
\hline Mimosaceae & Calliandra selloi & & $X$ & & & \\
\hline Mimosaceae & Mimosa bimucronata & $X$ & $\mathrm{X}$ & $\mathrm{X}$ & $\mathrm{X}$ & \\
\hline Mimosaceae & Mimosa scabrella & $X$ & & & & \\
\hline Moraceae & Sorocea bonplandii & & $\mathrm{X}$ & $\mathrm{X}$ & & \\
\hline Oleaceae & Ligustrum sinense & & & & & $\mathrm{X}$ \\
\hline Piperaceae & Piper guadichaudianum & & $\mathrm{X}$ & $\mathrm{X}$ & & \\
\hline Rubiaceae & Chomelia obtusa & & $\mathrm{X}$ & $\mathrm{X}$ & & \\
\hline Rubiaceae & Psychotria carthagenensis & & $\mathrm{X}$ & $\mathrm{X}$ & & \\
\hline Ulmaceae & Celtis iguanaea & & $\mathrm{X}$ & & $\mathrm{X}$ & \\
\hline Ulmaceae & Trema micrantha & & $\mathrm{X}$ & $\mathrm{X}$ & & \\
\hline
\end{tabular}

\subsection{Descrições dos Esporomorfos}

\section{PTERIDOPHYTA}

\section{Blechnaceae}

Blechnum meridense (K1.) Mett.

Figura $2 \mathrm{ab}$.

Mônades, esporos grandes, âmbito elipsoidal, reniformes, monoletes, exósporo psilado. $\mathrm{O}$ perisporo não foi considerado parte do exósporo. P: 34 um (2444), E: $58 \mu \mathrm{m}$ (49-70) e esporoderme: $2,7 \mu \mathrm{m}(1,5-4)$.

Material examinado: HERULBRA 1359. Arroio Piaí, Caxias do Sul, RS, Brasil, S. Diesel 459 (19.03.1998).

\section{Schizeaceae}

Anemia phyllitidis (L.) Sw.

Figura $2 \mathrm{~cd}$.

Mônades, esporos grandes, âmbito triangular, subprolatos, triletes, exósporo densamente baculado. Báculas sobre muros, distribuídas uniformemente sobre os esporos e unidas por suas bases. P: $92 \mu \mathrm{m}$ (74-109), E: $74 \mu \mathrm{m}$ (60-97), espoderme: $3 \mu \mathrm{m}$ (2-4) e báculas: 11 $\mu \mathrm{m}(8-13)$
Material examinado: HERULBRA 2517. Rio Caí, estrada para Vila Oliva, Gramado, RS, Brasil, S. Diesel 1617 (24.03.2001).

\section{ANGIOSPERMAE}

\section{Berberidaceae}

Berberis laurina Thunb.

Figura 2e-f.

Mônades, grãos de pólen médios, âmbito circular, esféricos, espiroaperturados, exina microrreticulada. D: $43 \mu \mathrm{m}$ (36-55), Ex: 2,2 $\mu \mathrm{m}$ (1-3) e O: < $1 \mu \mathrm{m}$.

Material examinado: HERULBRA 1095. Rio do Pinto, São Francisco de Paula, RS, Brasil, S. Diesel 195 (13.12.1997).

\section{Caprifoliaceae}

Sambucus australis Cham. \& Schltdl.

Figura $2 \mathrm{~g}-\mathrm{i}$.

Mônades, grãos de pólen pequenos, âmbito subtriangular, subprolatos, tricolporados,

R. Árvore, Viçosa-MG, v.33, n.5, p.895-905, 2009 

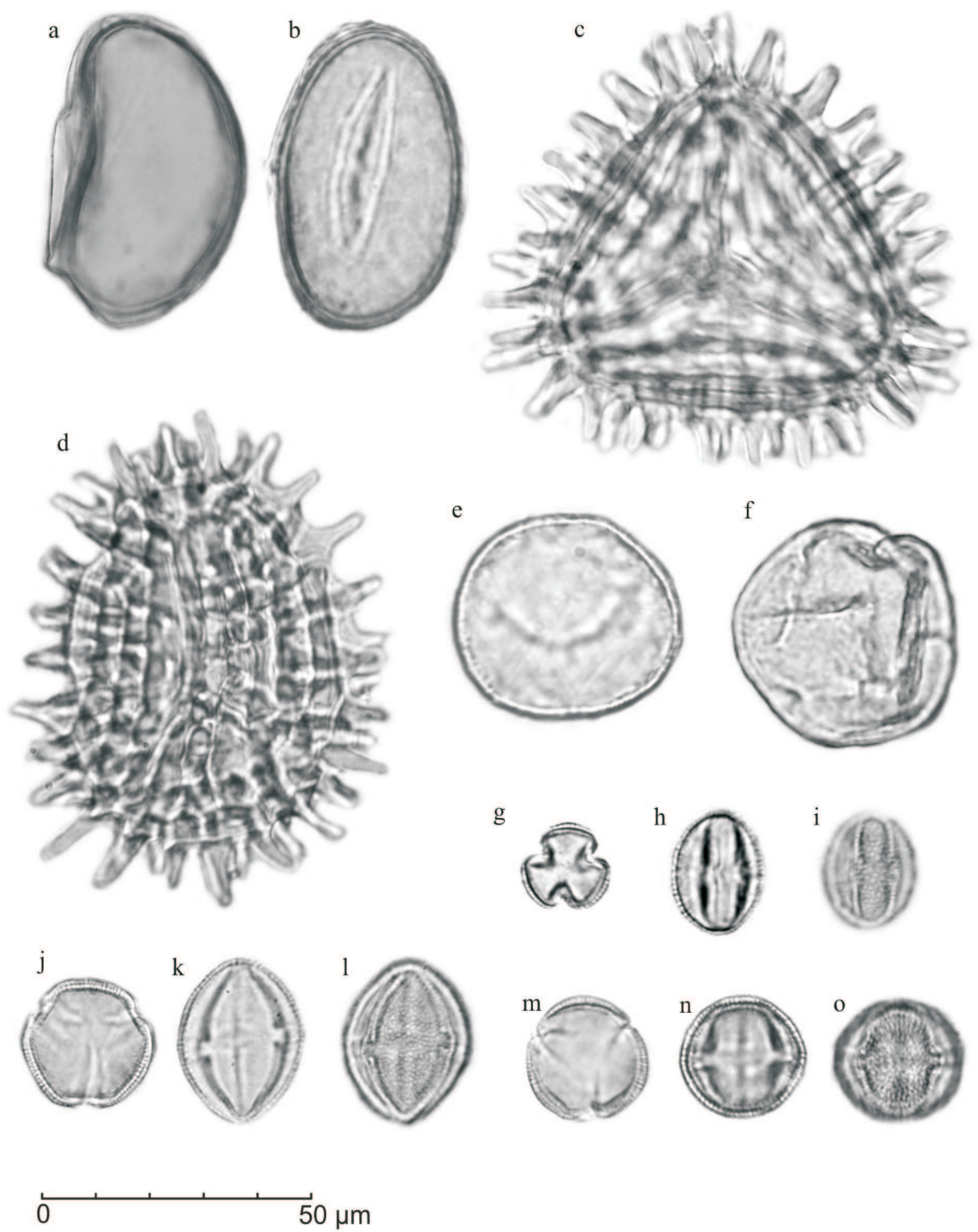

Figura 2-Grãos de pollen a-b. Blechmum meridense: a. VE, b. face proximal; c-d. Anemia phyllitidis: c. VP, d. VE; ef. Berberis Laurina: e. VP, f. vista lateral; g-i. Sambucus australis: g. VP, h. VE, i. detalhe da ornamentação; j-1. Erytroxylum argentinum: j. VP, k. VE, 1. detalhe da ornamentação; m-o. Maytenus ilicifolia: m. VP, n. VE, o. detalhe da ornamentação.

Figure 2 - Pollen grains a-b. Blechnum meridense: $a$. EV, b. proximal face; $c$-d. Anemia phyllitidis: $c . P V, d . E V ;$; $-f$. Berberis laurina: e. PV, f. side view; g-i. Sambucus australis: $g . P V, h . E V$, i. details of ornamentation; $j-1$. Erytroxylum argentinum: $j . P V, k . E V, 1$. details of ornamentation; $m-o$. Maytenus ilicifolia: $m$. PV, $n$. EV, $o$. details of ornamentation.

R. Árvore, Viçosa-MG, v.33, n.5, p.895-905, 2009 
endoabertura lalongada, exina reticulada com lumens distanciados. P: $22 \mu \mathrm{m}(20-25), \mathrm{E}: 17 \mu \mathrm{m}$ (13-20), Ex: $2,0 \mu \mathrm{m}(1-3)$ e $\mathrm{O}:<1 \mu \mathrm{m}$.

Material examinado: HERULBRA 966. Arroio Pinhal, Galópolis, Caxias do Sul, RS, Brasil, S. Diesel 66 (24.09.1997).

\section{Celastraceae}

Maytenus ilicifolia Mart. ex Reiss.

Figura 2m-o.

Mônades, grãos de pólen pequenos, âmbito subtriangular, esféricos, tricolporados; exina reticulada, homobrochada. P: $23 \mu \mathrm{m}$ (20-26), E: 23 $\mu \mathrm{m}$ (20-29), E: $1 \mu \mathrm{m}$ e O: 1,9 $\mu \mathrm{m}$ (1-2).

Material examinado: HERULBRA 1108. Rio do Pinto, São Francisco de Paula, RS, Brasil, S. Diesel 208 (13.12.1997).

\section{Ericaceae} G.Don

Agarista eucalyptoides (Cham. \& Schltdl.)

Figura 3a-c.

Tétrade tetraédrica, calimada e coaperturada, em que os grãos de pólen contíguos têm aberturas unidas formando pares em seis pontos da tétrade, endoabertura lalongada, exina escabrada. D: 42 $\mu \mathrm{m}$ (36-50), Ex: 2,6 $\mu \mathrm{m}$ e O: $<1 \mu \mathrm{m}$.

Material examinado: HERULBRA 1836. Rio Santa Cruz, Parque da Cachoeira, Canela, RS, Brasil, S. Diesel 936 (19.11.1998).

\section{Erythroxylaceae}

Erythroxylum argentinum O. E. Schulz

Figura 2j-1.

Mônades, grãos de pólen médios, âmbito subtriangular, subprolatos, tricolporados; endoabertura lalongada; exina reticulada, homobrochada. P: $30 \mu \mathrm{m}$ (26-38), E: $25 \mu \mathrm{m}$ (20-29), Ex: $2 \mu \mathrm{m}$ e $\mathrm{O}:<1 \mu \mathrm{m}$.
Material examinado: HERULBRA 3302. Arroio do Ouro, Vale Real, RS, Brasil, S. Diesel 737 (15.10.1998).

\section{Escalloniaceae}

Escallonia megapotamica Spreng.

Figura 3d-e.

Mônades, grãos de pólen pequenos, âmbito subtriangular, subprolatos, tricolporados; colpos com margens espessas, endoabertura lalongada e pouco evidente; exina psilada. P: $20 \mathrm{~mm}$ (18-23), E: $16 \mathrm{~mm}(14-21)$ e Ex: 2,1 mm (1-3).

Material examinado: HERULBRA 1100. Rio do Pinto, São Francisco de Paula, RS, Brasil, S. Diesel 200 (13.12.1997).

\section{Flacourtiaceae}

Casearia sylvestris $\mathrm{Sw}$.

Figura 3 fg.

Mônades, grãos de pólen médios, âmbito subtriangular, prolatos, tricolporados; colpos longos; área polar pequena; endoabertura lalongada provida de costa; exina psilada. P: $30 \mu \mathrm{m}$ (25-33), E: $22 \mu \mathrm{m}(18-25)$ e Ex: 2,0 $\mu \mathrm{m}(1,5-2)$.

Material examinado: HERULBRA 969. Arroio Pinhal, Galápolis, Caxias do Sul, RS, Brasil, S. Diesel 69 (24.09.1997).

\section{Melastomataceae}

Miconia hyemalis A.St.-Hil. \& Naudin ex Naudin

Figura 3 hi.

Mônades, grãos de pólen pequenos, âmbito circular, prolatoesferoidais, heterocolpados (três colpos com nós lalongados e três pseudocolpos), exina escabrada. P: $21 \mu \mathrm{m}$ (16-23), E: $19 \mu \mathrm{m}$ (1623), Ex: $2 \mu \mathrm{m}$ e O: $<1 \mu \mathrm{m}$.

R. Árvore, Viçosa-MG, v.33, n.5, p.895-905, 2009 


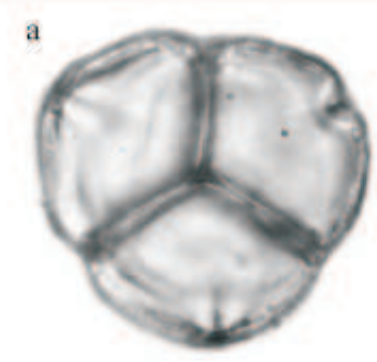

b
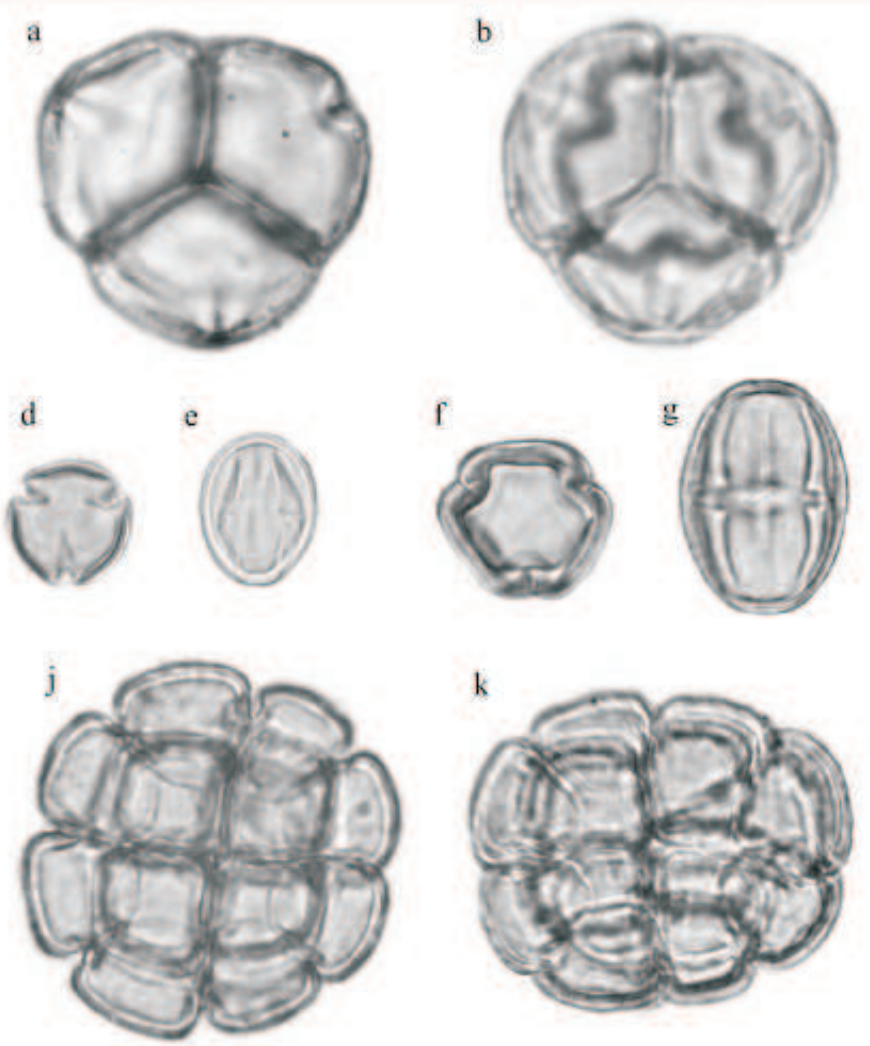

p

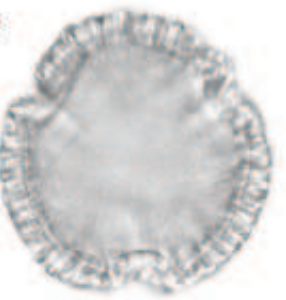

f

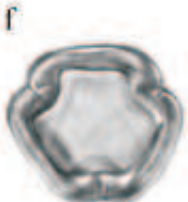

k

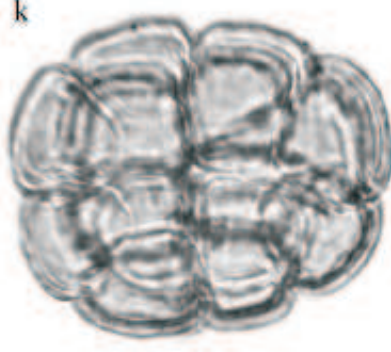

q

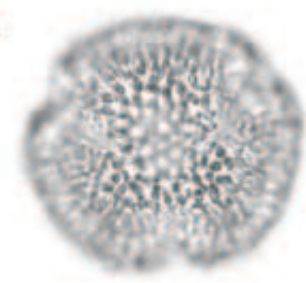

c

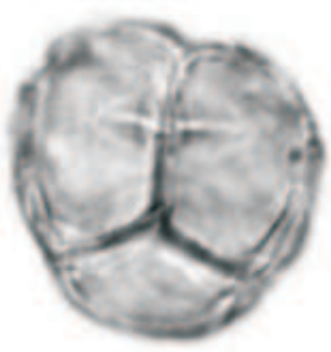

$\mathrm{g}$

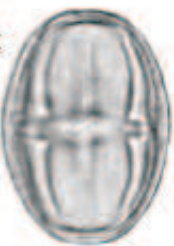

h

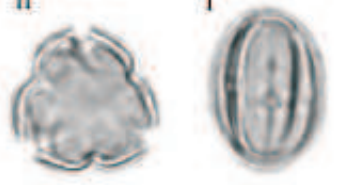

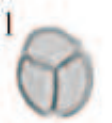

m.
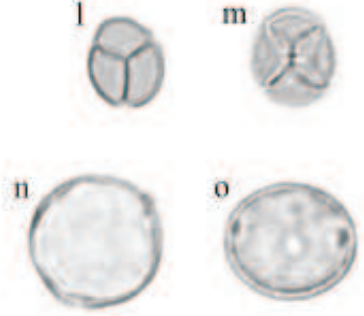

r

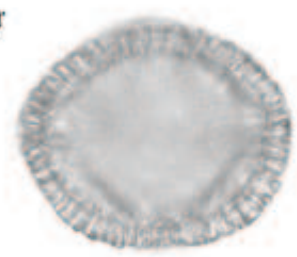

s
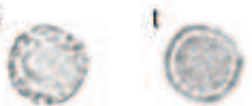

u

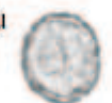

Figura 3 - Grãos de pollen a-c. Agarista eucalyptoides: a. vista frontal em primeiro plano, b, vista frontal e segundo plano, c. detalhe da co-apertura; d-e. Escallonia megapotamica: d. VP, e. VE; f-g. Casearia silvestres: f. VP, g. VE; h-i. Miconia hyemalis: h. VP, i. VE; j-k. Mimosa bimucronata: j. vista frontal, k. vista latera; 1-m. M. scabrella: 1. vista frontal em primeiro plano, m. vista frontal em segundo plano; n-o. Sorocea bomplandit: n. VP; o. VE; p-r. Ligustrum sinense: p. VP, q. detalhe da ornamentação, r. VE; s-u. Peperomia megapotamica: s. VP, t. detalhe da ornamentação, u. VE.

Figure 3 - Pollen grains a-c. Agarista eucalyptoides: a. front view in the foregrond, $b$. front view in the background, $c$. details of the co-apertured; $d$-e. Escallonia megapotamica: d. PV, e EV; f-g. Casearia silvestres: $f . V P, g . V E$; $h$-i. Miconia hyemalis: $h$. VP, $i$. VE; j-k. Mimosa bimucronata: j. front view, $k$. side view; $1-m$. M. scabrella: 1. front view in the foreground, $m$. ront view in the background; $n$-o. Sorocea bomplandit: $n$. PV; $o$. EV; $p$ r. Ligustrum sinense: $p$. PV, q. details of ornamentation, $r$. EV; $s$-U. Peperomia megapotamica: s. PV, t. details of ornamentation, $u$. EV.

R. Árvore, Viçosa-MG, v.33, n.5, p.895-905, 2009 


\section{Mimosaceae}

Calliandra selloi J.F.Macbr.

Figura 4a.

Políade muito grande, oito células em bi-tétrade, forma alongada, sendo uma extremidade arredondada e a outra cônica com um poro terminal, acalimada; exina rugulada. DM: $160 \mu \mathrm{m}$ (139-185), Dm: $96 \mu \mathrm{m}$ (81-115), Ex: 2,5 $\mu \mathrm{me}$ $\mathrm{O}:<1 \mu \mathrm{m}$.

Material examinado: HERULBRA 972. Arroio Pinhal, Galápolis, Caxias do Sul, RS, Brasil, S. Diesel 72 (24.09.1997).

\section{Mimosa bimucronata Kuntze}

Figura $3 j k$.

Políade com 16 grãos de pólen, circular em vista frontal e elíptica em vista lateral, acalimada. Grãos de pólen triporadopos, com três poros na face distal; exina escabrada. D: $45 \mu \mathrm{m}$ (39-51), Ex: $2 \mu \mathrm{m}$ e O: $<1 \mu \mathrm{m}$.

Material examinado: HERULBRA 1076. RioSantaCruz, Canela, RS, Brasil, S. Diesel 176(10.12.1997).

Mimosa scabrella Benth.

Figura $31 \mathrm{~m}$.

Tétrade pequena, cruzada, de contorno oval, acalimada. Grãos de pólen triporados, com três poros na face distal; exina escabrada. DM: $13 \mu \mathrm{m}$ (12-14), Dm: $9 \mu \mathrm{m}$ (8-10), Ex: $1 \mu \mathrm{m}$ e O: $<1 \mu \mathrm{m}$.

Material examinado: HERULBRA 1504. RioSantaCruz, Parque da Cachoeira, Canela, RS, Brasil, S. Diesel 604 (10.08.1998).

\section{Moraceae}

Sorocea bonplandii (Baill.) W.C.Burger, Lanj. \& Bôer

Figura 3n-o.

Mônades, grãos de pólen pequenos, âmbito subcircular, suboblatos, triporados ocasionalmente, di ou tetraporados; poros providos de áspide e operculados; exina granulada. P: $14 \mu \mathrm{m}$ (12-16), E: $18 \mu \mathrm{m}$ (16-21), Ex: $1 \mu \mathrm{m}$ e O: < $1 \mu \mathrm{m}$.

Material examinado: HERULBRA 943. Rio Caí, estrada para Vila Oliva, Gramado, RS, Brasil, S. Diesel43(07.09.1997).

\section{Oleaceae}

Ligustrum sinense Hott. ex Decne.

Figura 3p-r.

Mônades, grãos de pólen médios, âmbito subtriangular, suboblatos, tricolporados; endoabertura lalongada; exina retipilada. P: $29 \mu \mathrm{m}$ (27-30), E: $34 \mu \mathrm{m}$ (31-36), Ex: 1,9 $\mu \mathrm{m}$ $(1,5-2,0)$ e O: $3,0 \mu \mathrm{m}(2,5-3,0)$.

Material examinado: HERULBRA 1662. Rio Santa Cruz, Parque da Cachoeira, Canela, RS, Brasil, S. Diesel $762(22.10 .1998)$.

\section{Piperaceae}

Peperomia megapotamica Dahlst.

Figura $3 \mathrm{~s}-\mathrm{u}$.

Mônades, grãos de pólen pequenos, âmbito circular, subprolatos, monocolpados, ocasionalmente inaperturados; exina verrucada. P: $12 \mu \mathrm{m}$ (9-13), E: $10 \mu \mathrm{m}$ (9-12), Ex: 1 mm e $\mathrm{O}:<1 \mu \mathrm{m}$.

Material examinado: HERULBRA 952. Rio Caí, estrada para Vila Oliva, Gramado, RS, Brasil, S. Diesel52(07.09.1997).

Piper gaudichaudianum Kunth ex C.DC.

Figura 4bc.

Mônades, grãos de pólen pequenos, âmbito circular, prolatos, monocolpados; exina escabrada.

P: $14 \mu \mathrm{m}(11-16)$, E: $9 \mu \mathrm{m}$ (7-12), Ex: 1 mm e O:<1 $\mu \mathrm{m}$.

Material examinado: HERULBRA 1652. Arroio do Ouro, Vale Real, RS, Brasil, S. Diesel 752 (15.10.1998).

\section{Piper miquelianum DC.}

Figura 4de.

Mônades, grãos de pólen pequenos, âmbito circular, prolatos, monocolpados; exina escabrada.

P: $10 \mu \mathrm{m}(8-11), \mathrm{E}: 8 \mu \mathrm{m}$ (7-10), Ex: $1 \mathrm{~mm}$ e O: < $1 \mu \mathrm{m}$.

Material examinado: HERULBRA 921. Rio Caí, estrada para Vila Oliva, Gramado, RS, Brasil, S. Diesel 21 (25.08.1997).

R. Árvore, Viçosa-MG, v.33, n.5, p.895-905, 2009 

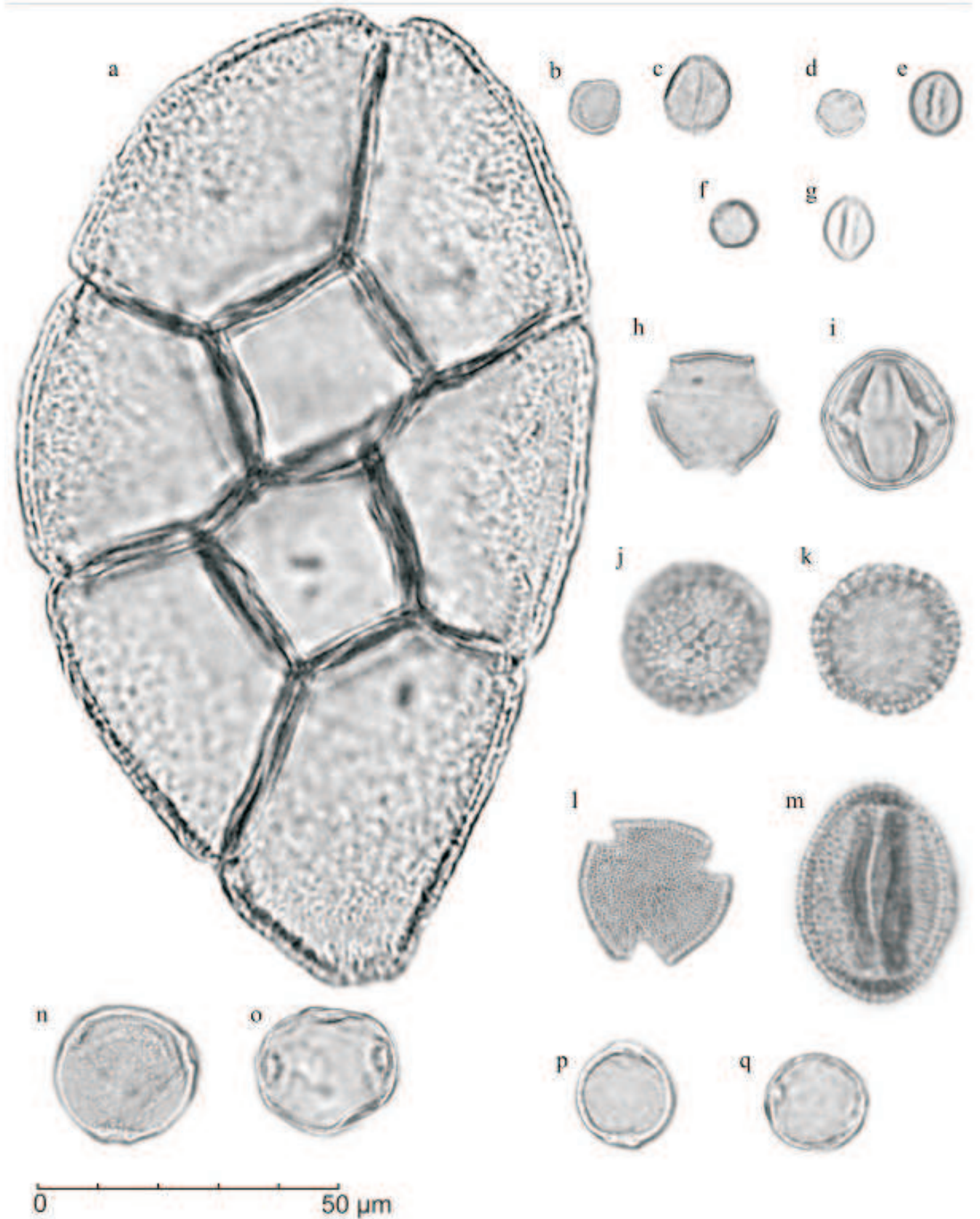

Figura 4 - Grãos de pollen a. Caliandra selloi: a vista frontal; b-c. Piper gaudichaudianum: b. VP, c. VE; d-e. P. miquelianum: d. VP, e. VE; f-g. Piper xylosteoides: f. VP, g. VE; h-i. Prumus myrtifolia: h. VP, i.VE; j-k. Chomelia obtusa: j. vista em primeiro plano, k. vista em segundo plano; I-m. Psychotria carthagenensis: I. VP; m. VE; n-o. Celtis iguanea: n. VP, o. VE; p-q. Trema micrantha: p. VP, q. VE.

Figure 4 - Pollen grains a, Caliandra selloi: a. front view; $b$-c. Piper gaudichaudianum: $b . V P, c$. VE; $d$-e. P. miquelianum: d. VP, e. VE; f-g. Piper xylosteoides: $f$. VP, g. VE; h-i. Prumus myrtifolia: $h$. VP, i.VE; j-k. Chomelia obtusa: $j$. view in the foreground $k$. view in the background; I-m. Psychotria carthagenensis:

R. Árvore, Viçosa-MG, v.33, n.5, p.895-905, 2009 
Piper xylosteoides Steud.

Figura 4 fg.

Mônades, grãos de pólen pequenos, âmbito circular, prolatos, monocolpados; exina escabrada.

P: $11 \mu \mathrm{m}(8-13)$, E: $9 \mu \mathrm{m}$ (7-11), Ex: 1 mm e O: < $1 \mu \mathrm{m}$.

Material examinado: HERULBRA 947. Rio Caí, estrada para Vila Oliva, Gramado, RS, Brasil, S. Diesel 47 (07.09.1997).

\section{Rosaceae}

Prunus myrtifolia (L.) Urb.

Figura 4h-i.

Mônades, grãos de pólen médios, âmbito subtriangular a circular, prolatoesferoidais, tricolporados; colpos com margem espessa e ornamentada; endoabertura lalongada; exina estriada com estrias mais evidentes nos polos. P: $25 \mu \mathrm{m}$ (22-28), E: $24 \mu \mathrm{m}$ (22-27), Ex: 2 mm e O: < $1 \mu \mathrm{m}$.

Material examinado: HERULBRA 1070. Arroio Piaí, Caxias do Sul, RS, Brasil, S. Diesel 170 (03.12.1997).

\section{Rubiaceae}

Chomelia obtusa Cham. \& Schltdl.

Figura $4 \mathrm{j}-\mathrm{k}$

Mônades, grãos de pólen médios, âmbito circular, esféricos, inaperturados, exina retipilada. P: $26 \mu \mathrm{m}$ (2428), E: $26 \mu \mathrm{m}$ (24-28), Ex: $2 \mu \mathrm{m}$ e O: $2 \mu \mathrm{m}$.

Material examinado: HERULBRA 3310. Afluente do Arroio Feitoria, Dois Irmãos, RS, Brasil, S. Diesel 2410 (09.10.2001).

Psychotria carthagenensis Jacq.

Figura $41 \mathrm{~m}$.

Mônades, grãos de pólen médios, âmbito subtriangular, prolatoesferoidais, tricolpados; exina reticulada, heterobrochada. P: $40 \mu \mathrm{m}$ (30-48), E: $37 \mu \mathrm{m}(32-42)$, Ex: $1,8 \mu \mathrm{m}(1-2)$ e O: $2 \mu \mathrm{m}$.

Material examinado: HERULBRA 1030. Arroio Pinhal, Galópolis, Caxias do Sul, RS, Brasil, S. Diesel 130 (29.11.1997).

\section{Ulmace a e}

Celtis iguanaea (Jacq.) Sarg.

Figura 4no.

Mônades, grãos de pólen pequenos, âmbito circular a subcircular, suboblatos, triporados; exina escabrada. P: $20 \mu \mathrm{m}$ (16-27), E: $24 \mu \mathrm{m}$ (21-29), Ex: 1,9 $\mu \mathrm{m}(1,5-2)$ e $\mathrm{O}:<1 \mu \mathrm{m}$.

Material examinado: HERULBRA 996. Arroio Pinhal, Galópolis, Caxias do Sul, RS, Brasil, S. Diesel 96 (24.09.1997).

\section{Trema micrantha (L.) Blume}

Figura 4pq.

Mônades, grãos de pólen pequenos, âmbito circular a subcircular, oblatoesferoidais, diporados, ocasionalmente triporados; exina escabrada. P: $17 \mu \mathrm{m}$ (15-20), E: $18 \mu \mathrm{m}$ (14-21, Ex: $1 \mu \mathrm{m}$ e O: < $1 \mu \mathrm{m}$.

Material examinado: HERULBRA 1012. Rio Caí, Vila Cristina, Caxias do Sul, RS, Brasil, S. Diesel 112 (15.11.1997).

\section{DISCUSSÃO E CONCLUSÕES}

Entre os 24 grãos de pólen e esporos descritos e ilustrados, 23 são dados inéditos do Rio Grande do Sul, e somente Anemia phyllitidis foi anteriormente descrito por Lorscheitter et al. (1998). Tal fato demonstra a carência de dados sobre estudos polínicos para o Estado, além de motivar esforços para a implementação de coleções que contituem o primeiro passo para a elaboração de inventários da biodiversidade.

Alguns táxons mostraram-se importantes devido à sua especificidade vegetacional, como no caso de Berberis laurina, Miconia hyemalis e Mimosa scabrella, que só ocorrem na Floresta Ombrófila Mista; Sambucus australis e Calliandra selloi, pois têm sua ocorrência preferencial na Floresta Estacional Decidual; e Chomelia obtusa, Sorocea bonplandii, Piper guadichaudianum, Psychotria carthagenensis e Trema micrantha estão vinculados às Florestas Estacional e Estacional Semidecidual; Escallonia megapotamica é comumente encontrada em ambientes florestais, mas ocorre indiscriminadamente na Floresta Ombrófila Mista,

R. Árvore, Viçosa-MG, v.33, n.5, p.895-905, 2009 
Floresta Estacional Decidual e Floresta Estacional Semidecidual. À Ligustrum sinense, por ser elemento exótico à flora local, pode ser atribuída uma valoração na interpretação ambiental, pois indica locais com interferência humana.

Estudos de palinologia atual fornecem suporte para a identificação dos principais conjuntos polínicos das comunidades vegetacionais, para entendimento de seu modo de dispersão e fluxo gênico entre as populações. Juntamente com outros fatores, esses dados subsidiam a tomada de decisões em planos de manejo ambiental, o qual envolve resoluções com base em complexas interações de fatores bióticos e abióticos (LACHOWSKI et al., 1994).

As florestas tropicais vêm sofrendo com a crescente onda de desmatamento, desenvolvimento urbano e conversão de áreas para silvicultura e agropecuária (RIBEIRO et al., 2005). E a identificação dos conjuntos polínicos fósseis por similaridade aos modernos pode fornecer pistas para o entendimento da história vegetacional da região em estudo. $\mathrm{O}$ conhecimento desses dados e a sua utilização em manejo de paisagens poderão auxiliar a formação de florestas produtivas com menores riscos ao ambiente e maior manutenção da biodiversidade (FERRAZ e VETTORAZZI, 2003).

\section{AGRADECIMENTOS}

À Profa. Dra. Suzete Diesel, pela cessão das exsicatas; ao Prof. Dr. Sérgio de Augusto Loreto Bordignon, pela revisão da nomenclatura botânica; e aos biólogos Anabela Lenhart Lopes e Marcus V. G. Corrêa, pelo auxílio na coleta e processamento do material polinífero.

\section{REFERÊNCIAS}

BACKES, A.; NARDINO, M. Árvores, arbustos e algumas lianas nativas no Rio Grande do Sul. 2.ed. São Leopoldo: 2003. 213p.

B ARTH, O. M.; MELHEM, T. S. Glossário ilustrado de palinologia. Campinas: UNICAMP, 1988. 77p.

R. Árvore, Viçosa-MG, v.33, n.5, p.895-905, 2009
COLINVAX, P.; OLIVEIRA, P. E.; PATIÑO, J. E. M. Amazon pollen manual and atlas. Amsterdam: Harwood Academic Publishers, 1999. 332p.

DIESEL, S. Estudo Fitossociológico da vegetação da bacia hidrográfica do rio Caí Rio Grande do Sul. Leon: Universidad de León, 2005. 504p

ERDTMAn, G. Pollen morphology and plant taxonomy - Angiosperms. Waltham: The Chronica Botanica, 1952. 539p.

FERRAZ, S. F. B.; VETTORAZZI, C. A. Identificação de áreas para recomposição florestal com base em princípios de ecologia de paisagem. Revista Árvore, v.27, n.4, p.575-583, 2003.

\section{INTERNATIONAL PLANT NAMES}

INDEX - INPI. Disponível em:<www.ipni.org> Acesso em: 5 nov. de 2007.

LACHOWSKI, H. M. et al. Remote sensing and GIS: their role in ecosystem management. Journal of Forestry, v.92, n.8, p.39-40, 1994.

LORSCHEITTER, M. L. et al. Pteridophyte spores of Rio Grande do Sul flora, Brasil. Part I.

Palaeontographica, v.246, n.1-3, p.1-113, 1998.

LOPES, S. B.; GONÇALVES, L. Elementos para aplicação prática das árvores nativas do sul do Brasil na conservação da biodiversidade. $18 \mathrm{p}$. Disponível em: <www.fzb.rs.gov.br> Acesso em: 28 nov. 2007.

MELHEN, T. S. et al. Variabilidade polínica em plantas de Campos do Jordão (São Paulo, Brasil). Boletim do Instituto de Botânica, v.16, p16-104, 2003.

MISSOURI BOTANICAL GARDEN MOBOT. Disponível em: <www.mobot.org> Acesso em: 17 nov. de 2007.

NEVES, P. C. P.; BAUERMANN, S. G. Catálogo Palinológico de coberturas quaternárias no Estado do Rio Grande do Sul (Guaíba e Capão do Leão), Brasil. Descrições taxonômicas - Parte I: fungos, algas, palinomorfos outros e fragmentos de invertebrados. Pesquisas Série Botânica, v.53, p.121-149, 2003. Disponível em: <www.ulbra.br/ palinologia>.

\section{R. Árvore, Viçosa-MG, v.33, n.5, p.895-905, 2009}


NEVES, P. C. P.; BAUERMANN, S. G. \& KRÖEFF, V. N. Catálogo Palinológico em coberturas quaternárias no Estado do Rio Grande do Sul (Guaíba e Capão do Leão), Brasil. Descrições taxonômicas - Parte III:

Magnoliophyta (Liliopsida) e Gymnospermae.

Acta Geológica Leopoldensia, v.56, n.1, p.35-45, 2003. Disponível em: <www.ulbra.br/ palinologia>.

PUNT, W. et al.. Glossary of pollen and spore terminology. 2007. Disponível em: <www.biol.ruu.nl./ palaeo/glossary/> Acesso em: 20 dez. de 2007.

RIBEIRO, C. A. A. S. et al. O desafio da delimitação de áreas de preservação permanente. Revista Árvore, v.29, n.2, p.203-212, 2005.

Roubik, D. W.; MOReno, J. E. Pollen and spores of Barro Colorado Island. St. Louis, Missouri Botanical Garden, 270p.1991. (Monographsin Systematic Botany, v.36)
SALGADO-LABORIAU, M. L. Contribuição à palinologia dos cerrados. Rio de Janeiro: Academia Brasileira de Ciências, 1973. 291p.

SEPLAN/IBGE. Porto alegre e parte das folhas SH.21 Uruguaiana e SH.22 Lagoa Mirim: geologia, geomorfologia, pedologia, vegetação, uso potencial da terra. Rio de Janeiro: 1986. 796p. 6 mapas.

SOBRAL, M. et al. Flora arbórea e arborescente do Rio Grande do Sul, Brasil. São Carlos/Porto Alegre, 2006. 350p.

WILLARD, D. A. et al. Atlas of pollen and spores of the Florida Everglades. Palynology, v. 28, p.175-227, 2004.

WILlberGer, T. P. et al. Flora do Setor Oriental do Planalto Sul-Rio-

Grandense. Guia de espécies vegetais. São Leopoldo: ALPP, 2004. v.1. p.59. Disponível em: <www.ulbra.br/palinologia>. 